\title{
Protection to Six Vaccinable Pathogens in Paired Plasma/DBS and HIV Impact in Infected and Uninfected Children From Kinshasa
}

\author{
Rodríguez-Galet Ana \\ Ramón y Cajal University Hospital-IRYCIS \\ Rubio-Garrido Marina \\ Ramón y Cajal University Hospital-IRYCIS \\ Valadés-Alcaraz Ana \\ Ramón y Cajal University Hospital-IRYCIS \\ Rodríguez-Domínguez Mario \\ Ramón y Cajal University Hospital-IRYCIS \\ Galán Juan Carlos \\ Ramón y Cajal University Hospital-IRYCIS \\ Ndarabu Adolphe \\ Monkole Hospital. \\ Reina Gabriel \\ University of Navarra Clinic-IdiSNA \\ Holguín Africa ( $\square$ africa.holguin@salud.madrid.org ) \\ Ramón y Cajal University Hospital-IRYCIS
}

\section{Research Article}

Keywords: paediatric HIV, Kinshasa, vaccine-preventable diseases, dried blood samples (DBS), immunization coverage, low-income countries

Posted Date: November 8th, 2021

DOI: https://doi.org/10.21203/rs.3.rs-1019015/v1

License: @) (1) This work is licensed under a Creative Commons Attribution 4.0 International License. Read Full License 


\section{Abstract}

Child vaccination reduces infant mortality rates. HIV-infected children present higher risk of diseases than non-infected. We report the protection coverage rates to 6 vaccine-preventable diseases in a paediatric population from the Democratic Republic of the Congo (DRC) and the impact of HIV infection, providing the first data on the validity of dried blood samples (DBS) to monitor the immune protection. During 2016-2018 DBS from 143 children/adolescents were collected in Kinshasa (DRC), being 52 HIV-infected. Forty-two had a paired plasma sample. Protective IgG was quantified (VirClia ${ }^{\circledR} \mathrm{IgG}$,Vircell) to obtain the optimal cut-off in IgG detection in DBS. ROC curves were generated with R software and statistical analyses with Stata. Protective IgG levels varied across pathogens, not reaching the herd immunity. HIV-infected presented lower vaccine protection than uninfected for all analysed pathogens, except rubella, with statistically significant differences for measles $(30.8 \%$ vs. $53.8 \%$; $=0.008)$ and tetanus $(3.8 \%$ vs. $22 \%$; $=0.004)$. New cut-offs were calculated when using DBS to improve test performance. We reinforce the necessity to increase pediatric vaccination coverage in Kinshasa, specially in HIV seropositive, with less capacity to maintain adequate antibody levels. DBS were useful to monitor vaccination coverage in seroprevalence studies in resource-limited settings, after optimizing the cut-off value for each pathogen.

\section{Key Points}

In the article that we present bellow, we wanted to observe the protective IgG levels to six diseases in clindren from Kinshasa (2016-2018). These levels varied across pathogens, not reaching herd immunity. Immunization was lower among HIV-infected patients for all analysed pathogens except rubella. DBS can be useful to monitor seroprotection coverage when serum/plasma is absent. Cut-off values should be optimized when DBS are used for seroprevalence studies.

\section{Introduction}

Child vaccination is the most cost-effective public health intervention available to reduce the morbi-mortality in children and adolescents [1]. In the last three decades vaccination coverage against vaccine-preventable infectious diseases have increased, reducing the deaths by almost $60 \%$ from $1990-2019$; however, there are still 20 million children in the world who do not receive essential vaccines such as diphtheria-tetanus-pertussis-containing vaccine (DTP) or measles vaccine. A 39\% of them live in five countries: Democratic Republic of the Congo (DRC), Nigeria, India, Pakistan and Ethiopia [1], hosting 49\% of children's death under 5 years worldwide [2,3]. During 2019, approximately $85 \%$ of infants around the world received 3 doses of the DTP vaccine, used as an indicator of the ability of countries to provide immunization services [1].

The DRC presents high mortality rate in children under 5 years, reaching 85 per 1000 births [4]. According to the Multiple Indicator Cluster Survey, $65 \%$ of children under 2 years in the DRC had not received any vaccine or were incompletely vaccinated during 2018-2019 [5,6]. The absence of correct immunity in the paediatric population causes periodic outbreaks of vaccine-preventable diseases in the country [7].

According to 2020 WHO data for the DRC, the coverage of DTP third doses (DTP3) and measles-containing-vaccine (MCV) was $57 \%$, with no data of rubella vaccine coverage. The DRC vaccination schedule does not yet contemplate the second dose of MCV [8], used to assess the ability to continue immunization services between the second and fifth year of life [3].

Children living with the human immunodeficiency virus (HIV) tend to have lesser vaccine protection against vaccine-preventable diseases vs. unexposed children [9]. Thus, it is important to optimize the vaccination schedule, especially in countries with a high rate of paediatric HIV infections. Protective titters after vaccine immunization in HIV-infected children varies depending on the vaccine, immune recovery and viral suppression [10]. Revaccination after initiating antiretroviral therapy (ART) could help to increase protection in HIV-infected children [11,12]. Most of them live in low-middle income countries, where scarce laboratory resources and limited conditions for collecting and handling serum/plasma from blood difficult monitoring of real paediatric vaccination coverage. In these circumstances, dried blood samples (DBS) could represent a convenient sample for antibody detection [13], being easy to collect, store and transport without cold chain [14]. DBS have been used in the surveillance and monitoring of numerous infectious diseases [13-19]. The good correlation between antibody concentrations in DBS and serum/plasma samples support the wider use of DBS in post-vaccination and seroepidemiological studies [13].

Our study reports the immune seroprotection rates to 6 vaccine-preventable diseases (diphtheria, tetanus, pertussis, measles, mumps, rubella) in a paediatric and adolescent population in Kinshasa (DRC), analysing the impact of HIV infection. Furthermore, we provide the first data on the validity of DBS to test the immune protection to these 6 pathogens, establishing the cut-off values that provide optimal sensitivity and/or specificity in DBS for each infection in the study cohort.

\section{Methods}

\section{Clinical and laboratory procedures}

DBS from 143 children and adolescents under clinical follow-up in Monkole and Kalembelembe hospitals (Kinshasa,DRC) were collected between 2016-2018, as previously described [20]. Forty-two of them (29.4\%) also had paired plasma. All specimens had associated clinical-epidemiological data. The samples were stored in Kinshasa hospitals at $-20^{\circ} \mathrm{C}$ until their transport to Madrid, where they were kept at $-80^{\circ} \mathrm{C}$ until processing to analyse and quantify the presence of protective lgG against six pathogens responsible for vaccine-preventable diseases: diphtheria, tetanus, pertussis, measles, mumps and rubella.

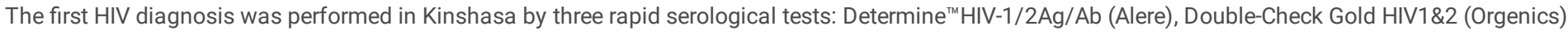
and Uni-Gold HIV (Trinity Biotech) in patients older than 18 months of age. In infants under 18 months, the 4th generation immunoassay VIDAS ${ }^{8}$ HIV Duo Ultra (Biomerieux) or, exceptionally, the molecular test Abbott real-time HIV-1 Qualitative were used. 
In the HIV-1 Molecular Epidemiology Laboratory (Madrid), the serological status of the children was confirmed by the Geenius ${ }^{\mathrm{TM}} \mathrm{HIV}-1 / 2$ (BioRad) assay. One

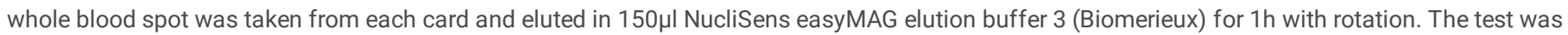
performed according to manufacturer indications, but using $40 \mu \mathrm{l}$ of the eluted, as previously published by our group [19]. The results obtained by

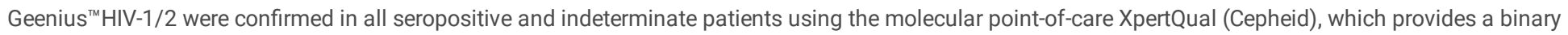
"detected"/"not detected", according to manufacturer's instructions.

\section{Quantification of IgG against six vaccinable diseases}

VirClia ${ }^{\circledR} \operatorname{lgG}($ Vircell) technique was used to quantify the presence of protective lgG against the six pathogens with $5 \mu$ l of plasma. When using DBS, one dot (70 $\mathrm{\mu l}$ of whole blood containing $42,7 \mu \mathrm{l}$ plasma after considering $39 \%$ haematocrit [21]) was eluted in $770 \mu \mathrm{l}$ of phosphate-buffered saline (PBS), being tested $100 \mu \mathrm{l}$ of the final elution in each specific VirClia ${ }^{\circledR} \lg \mathrm{G}$ test (equivalent to $5 \mu$ l plasma per test).

We considered as gold standard the cut-off values provided by VirClia ${ }^{\circledR}$ for the six pathogens in plasma/serum (Table 1), which provides $96-100 \%$ sensitivity and $100 \%$ specificity.

Table 1

VirClia IgG (Vircell) cut-off values for plasma/serum.

\begin{tabular}{|c|c|c|c|c|c|c|}
\hline & \multicolumn{3}{|c|}{ Semi-quantitative (IU/ml) } & \multicolumn{3}{|c|}{ Qualitative (index) } \\
\hline & Pertussis & Diphtheria & Tetanus & Measles & Mumps & Rubella \\
\hline \multirow[t]{3}{*}{ Positive } & $>120$ & $\geq 0,1$ & $>0,2$ & 1.1 & 1.1 & 1.1 \\
\hline & & Max. protection & & & & \\
\hline & & 0.01-0.09 Basic protection & & & & \\
\hline Indeterminated & $60-120$ & - & $0.1-0.2$ & $0.9-1.1$ & $0.9-1.1$ & $0.9-1.1$ \\
\hline Negative & $<60$ & $<0.01$ & $<0.1$ & $<0.9$ & $<0.9$ & $<0.9$ \\
\hline
\end{tabular}

To optimize the DBS use for IgG measurements, the sensitivity, specificity and positive (PPV) and negative (NPV) predictive value of the tests were calculated for each of the six measurements in DBS of the 42 children and adolescent under study with paired plasma/DBS, considering as reference the results obtained in plasma samples. To obtain an optimal cut-off that offered the maximum sensitivity and/or specificity in DBS for the detection of protective IgG against each pathogen, ROC curves were generated with R software. Indeterminate values were excluded from the final calculations. Statistical analyses were carried out with Stata.

\section{Ethical considerations}

The project was approved by the Human Subjects Review Committees at Monkole Hospital/University of Kinshasa (DRC) and Hospital Ramón y Cajal (Madrid, Spain). Informed consent was obtained from parents or guardians of enrolled participants. Children and adolescents also provided assent after parental consent when they could understand the meaning of participation in the study. All methods were carried out in accordance with relevant guidelines and regulations. Patients' names were codified at sampling to maintain confidenciality.

\section{Results}

\section{Study population}

The median age of the 143 patients was eleven years old, and $52.4 \%$ were male. Among them, 52 (36.4\%) were HIV-infected, most (80.8\%) presented viral load (VL) above $1000 \mathrm{cp} / \mathrm{ml}$, and only half (52.4\%) were ART experienced at sampling (Table 2 ). 
Table 2

Characteristics of the study population from Kinshasa (DRC).

\begin{tabular}{|c|c|c|c|}
\hline & $\begin{array}{l}\text { With paired DBS/plasma } \\
(\%)\end{array}$ & $\begin{array}{l}\text { Only with DBS } \\
\text { (\%) }\end{array}$ & Total \\
\hline Total available samples & $42(100)$ & $101(100)$ & $143(100)$ \\
\hline Male gender & $19(45.2)$ & $56(55.4)$ & $75(52.4)$ \\
\hline Median age at sampling [IQR] & 14 [12-17] & 9 [3-12.8] & $11[5-14]$ \\
\hline$<1$ & $1(2.4)$ & $18(17.8)$ & $19(13.3)$ \\
\hline$\geq 1-5$ & 0 & $12(11.9)$ & $12(8.4)$ \\
\hline$>5-10$ & 5 (11.9) & $22(21.8)$ & $27(18.9)$ \\
\hline$>10-15$ & $16(38.1)$ & $32(31.7)$ & $48(33.5)$ \\
\hline$>15$ & $18(42.9)$ & $16(15.8)$ & $34(23.8)$ \\
\hline Unknown & $2(4.7)$ & $1(1)$ & $3(2.1)$ \\
\hline \multicolumn{4}{|l|}{ Confirmed HIV status } \\
\hline HIV+ & $38(90.5)$ & $14(13.9)$ & $52(36.4)$ \\
\hline HIV- & $4(9.5)$ & $87(86.1)$ & $91(63.6)$ \\
\hline \multicolumn{4}{|l|}{ ARV experience and VL } \\
\hline ARV experience & $39(92.9)$ & $23(22.8)$ & $75(52.4)$ \\
\hline$\geq 1000 \mathrm{cp} / \mathrm{ml}$ & $31(73.8)$ & $11(10.9)$ & $42(29.4)$ \\
\hline \multicolumn{4}{|l|}{ Mother's HIV status } \\
\hline HIV+ & $17(40.5)$ & $43(42.6)$ & $60(41.9)$ \\
\hline HIV- & $4(9.5)$ & $24(23.7)$ & $28(19.6)$ \\
\hline Unknown & $21(50)$ & $34(33.7)$ & $55(38.5)$ \\
\hline
\end{tabular}

\section{Immunization level in plasma of the paediatric population.}

Protective IgG levels greatly varied for each pathogen in the study cohort. Different protection coverage was found in the 42 children and adolescents with available plasma for rubella (93\%), diphtheria (71\%), mumps (69\%), measles (64\%), tetanus (7\%) and pertussis (2\%) in plasma (Figure $1 \mathrm{a})$. Therefore, we found that $93 \%$ of children/adolescents did not show IgG protection against pertussis, $43 \%$ against tetanus, $29 \%$ against measles and $21 \%$ against mumps. The percentage of patients with indeterminate results in plasma was variable, ranging from $2 \%$ for rubella to $50 \%$ for tetanus (Figure $1 \mathrm{a}$ ). Indeterminate values were excluded from the final calculation.

\section{Cut-off calculation for each pathogen using DBS.}

To evaluate the validity of DBS to monitor the immune protection, we analysed the presence of protective lgG against the six pathogens in the 42 paired DBS/plasma specimens (Figure 1).

The sensitivity of each VirClia ${ }^{\circledR}$ IgG test for the detection of antibodies in DBS, considering the same cut-off for plasma (gold-standard sample) established by the manufacture's, was $100 \%$ for pertussis, diphtheria and mumps, $97.4 \%$ for rubella, lower for measles $(81.8 \%)$ and tetanus (66.7\%). Specificity was very high for pertussis $(97.6 \%)$, but decreased for tetanus ( $87.2 \%)$, measles $(40 \%)$, mumps $(7.7 \%)$, diphtheria and rubella (0\% each). The PPV ranged from $28.6 \%$ (tetanus) to $92.7 \%$ (rubella)

A new cut-off was calculated for each test, to achieve optimal sensitivity and/or specificity in the detection of IgG using DBS. The application of optimal cutoff for each pathogen allowed to increase the PPV and NPV of the tests from the study cohort when using DBS. (Table 3). 
Table 3

Results of VirClia ${ }^{\circledR}$ IgG test for the detection of protective IgG against six pathogens responsible for vaccine-preventable diseases using dried blood samples fr paediatric patients.

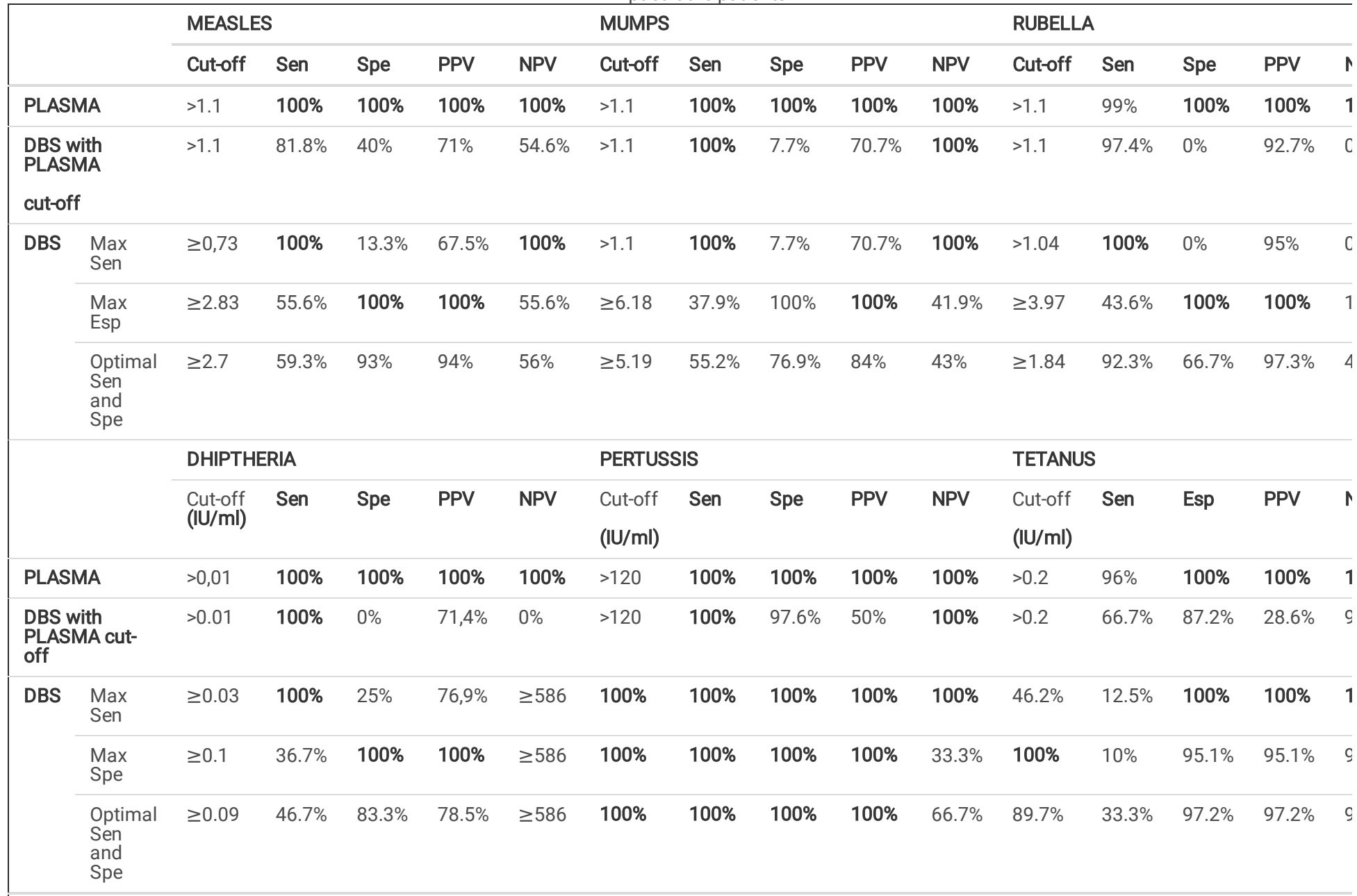

IU, international units; $\mathrm{ml}$, millilitre; PPV, positive predictive value; NPV, negative predictive value; DBS, dried blood spots sample; Max, maximum; Sen, sensitiv Spe, specificity. The cut-offs provided by VirCell for plasma have been taken as reference

\section{Comparison of immunization results in DBS vs. plasma in the 42 children and adolescents with paired samples.}

The agreement in the immune protection rate for the 6 pathogens under study in the 42 children with paired DBS/plasma specimens varied according to the considered cut-off value showed in Table 3.

When using the plasma cut-off for DBS, the percentage of immunized children in DBS for all pathogens was higher than when using plasma (Figure 2 options A vs. B), with significant differences for 2 of them ( $+28.6 \%$ for mumps and diphtheria). By contrast, the observed protection was only $+2.4 \%$, for pertussis, $+4.8 \%$ for rubella and $+9.5 \%$ for tetanus and measles.

When we applied in DBS the cut-off providing maximum (100\%) specificity (Figure 2 option C), the rate of protected children for all pathogens was underestimated from 2-3 fold times $v s$. values provided in plasma with VirClia cut-off for 5 pathogens: rubella (-50\%), diphtheria (-45.2\%), mumps (-42.9\%), measles $(-28.6 \%)$ and tetanus $(-4.8 \%)$. The only exception was pertussis, showing a similar low number of protected subjects than in plasma.

When the optimal cut-off showed in Table 3 for each pathogen was applied in DBS (Figure 2 option D), the percentage of immunized among these 42 children was lower than using plasma to diphtheria (-35.7\%), mumps and measles $(-23.8 \%)$ and rubella $(-4.8 \%)$, was slightly higher (+2.4\%) to tetanus, and similar to pertussis.

\section{Estimated immunization level in DBS of 143 children and adolescents.}

To study the protection coverage rates to 6 vaccine-preventable diseases in the whole study population from Kinshasa, we evaluated the percentage of children with protective IgG against each pathogen under study in the 143 DBS using cut-off providing maximum (100\%) specificity (corresponding to $0 \%$ false positives) described at Table 3. We decided to use this cut-off to guarantee that all patients identified as immunized in the whole study cohort were protected, avoiding overestimation in the percentage of immunized patients. We did not use the plasma cut-off due to the previously observed overestimation of the percentage of immunized for all pathogens (Figure 2 option B). 
With this $100 \%$ specificity cut-off, protective IgG were detected in less than half of the 143 studied patients: $46.9 \%$ for rubella, $45.5 \%$ for measles, $36.4 \%$ for diphtheria, $24.5 \%$ mumps, and $0.7 \%$ for pertussis. As it happened with the immunization values obtained when comparing the 42 paired samples DBS vs. plasma, immunization rates were between 2 and 3 times lower than those observed in plasma (gold standard) comparing data from the 143 DBS $v s .42$ plasma (15.4\% vs.7.1\%), except for tetanus, where the percentage of immunized patients doubled (Figure 3).

Impact of HIV infection in protection rate to 6 vaccine-preventable pathogens

Based on the analysis in the 143 DBS using the cut-off providing maximum (100\%) specificity (Figure 4A), we explored the impact of HIV infection in the protection coverage rates to the 6 vaccine-preventable diseases, protective lgG to each pathogen were measured in 52 HIV-infected vs. 91 uninfected children of the study population from Kinshasa. We observed lower vaccine protection in HIV infected children except for rubella, which was slightly higher. The differences were statistically significant for measles $(30.8 \%$ vs. $53.8 \%, \mathrm{p}=0.008)$ and for tetanus $(3.8 \%$ vs. $22 \%$, $\mathrm{p}=0.004)$. When the optimal cut-off was applied (Figure 4B) the significant differences between HIV-infected and uninfected were maintained in measles $(34.6 \%$ vs. $51.1 \%, p=0.001)$ and tetanus $(7.7 \%$ vs. $45.1 \%, \mathrm{p}=0.0001)$. However, a significant difference was also observed for rubella $(88.5 \%$ vs. $60.4 \%, \mathrm{p}=0.0001)$.

\section{Discussion}

The evaluation of immunization programs is key to identify areas of suboptimal vaccination coverage [22]. Our pilot study provides pioneer data related to the seroprotection to six infectious diseases preventable by vaccination in a cohort of children and adolescents living in Kinshasa (DRC) using samples collected during 2016-2018. Our results reveal that IgG levels greatly varied for each pathogen in the study population, not reaching the percentage necessary to achieve herd immunity. We also observed lower vaccine protection in HIV infected children vs. uninfected. We confirmed the usefulness of DBS in the absence of plasma/serum to study seroprevalence against vaccinable-preventive diseases to control vaccination coverage in resource-limited settings, after optimizing the cut-off value for each pathogen. We also provided the guideline for the adaptation of Vircell assays from serum to DBS, and the optimal pre-analytical treatment of DBS specimens for clinical vaccine or seroepidemiological studies.

Control of airborne viral infections with a very high reproduction number, like measles, mumps or rubella, requires high vaccine coverage [23,24]. Despite measles is endemic in the DRC, and $89-94 \%$ herd immunity is required to halt its transmission, coverage not reached in many African countries [25]. Among the 42 children and adolescents from Kinshasa with available plasma, 7 out of 10 were immunized against mumps and 9 out of 10 against rubella (Figure 2), despite the absence of both vaccines in the DRC vaccination schedule [26], revealing that protection was due to pathogen exposition and not by vaccination. We found that 6 out of 10 were immunized against measles, whose vaccine is administered at 9 months of age in the RDC.

Antibodies against measles, mumps and rubella can be present for up to 20 years after trivalent vaccine administration [27], decreasing in some children when the vaccines are given separately [28]. Although MMR vaccine-induced protection appears to persist at least into early adulthood, constant monitoring of protection against these pathogens is necessary [27]. In addition, it must be taken into account that the current vaccination schedule during the first year of life of older patients under study could differ from the current one given to younger participants, modifying the protection against some of the vaccine pathogens. Our data revealed similar immunization rates to measles (64\%) in the study paediatric population from Kinshasa during $2016-2018$ than in other paediatric cohorts in the DRC during 2013-2014 [29], being slightly higher than the vaccination coverage after the first and single dose of measles vaccine administered at 9 months of age reported by the WHO in that country (57\%) [8].

DTP3 coverage in the DRC, used as an indicator of the capacity of a country to provide immunization services, reached $57 \%$ in 2019 [8]. However, we found different immunization rates for each pathogen in the 42 plasma from the study population, being $71.4 \%$ of them immunized against diphtheria, but only $7.1 \%$ against tetanus and $2.4 \%$ against pertussis. If patients protected against diphtheria had suffered from the disease was not reported in the available clinical files. The high vulnerability to tetanus and pertussis of the Congolese paediatric and adolescent population is consistent with the latest official data from the country [8].

The interference with other infectious diseases affects the loss of seroprotection [30-34]. In this regard, we wanted to analyse the impact of HIV infection on seroprotection levels against the 6 pathogens in our paediatric cohort from Kinshasa. To avoid the loss of statistical robustness of the results due to the low sample size using plasma samples (only in 42 patients), we used DBS, the available sample in all 143 subjects under study. We considered the cut-off providing $100 \%$ specificity to guarantee that all patients identified as immunized were protected and to avoid overestimation in the rate of immunized children and adolescents in the complete study cohort. However, it is important to note that the cut-off providing $100 \%$ specificity in the detection of protective lgG for each pathogen in DBS might be always calculated in each study population with paired plasma/DBS samples and only be extrapolated to other population with similar prevalence for each analysed infection.

Since children living with HIV present a higher risk of vaccine-preventable infectious diseases than non-infected, vaccination with an adapted schedule, revaccination and periodic monitoring of their seroprotective status are recommended $[12,35,36]$. The low levels of seroprotection for all pathogens except rubella observed in the $52 \mathrm{HIV}$-infected patients vs. 91 HIV-uninfected could be explained by the lower capacity of the children with HIV to maintain the titters at adequate levels over time [35].

This study has several limitations. Firstly, plasma was only available in 42 of 143 subjects under study since we used retrospective and remaining samples from other studies in paediatric population in Kinshasa [20,37-39]. Furthermore, data related to immunization status and number of received doses per participant was not reported in the clinical files, preventing distinguish the children and adolescents with protective IgG due to vaccination or due to infection. Secondly, the number of positive/negative samples for each pathogen in our study population was very uneven among the 42 with available plasma specimens, ranging from only 1 subject infected with pertussis to 39 infected to rubella. Thus, future similar studies should include a higher number of subjects and a similar number of infected and uninfected children per infection, for a better adjustment of the cut-off values for each pathogen. Thirdly, the cut-off values providing $100 \%$ specificity in DBS for each pathogen could not be appropriate for other cohorts with different prevalence of the studied 
vaccinable-preventive diseases. Cut-off values providing $100 \%$ specificity should be always calculated in paired DBS/plasma specimens for the same setting or study cohort with a similar prevalence of vaccinable-preventive diseases under study. In addition, the provided new cut-offs for DBS would be Vircellspecific, and should not be extrapolated to other similar assays for seroprevalence studies.

\section{Conclusions}

Our data reinforce the necessity to increase/improve vaccination coverage in Kinshasa, paying special attention to the HIV-infected paediatric population, with less capacity to maintain antibody titters at adequate levels. To prevent future outbreaks and to avoid preventable deaths from vaccination, we emphasize the urgency for periodic monitoring immunity to vaccine-preventable diseases globally and mainly in countries with limited resources. The use of DBS could help to expand antibody detection for monitoring seroprotection level coverage among children in resource-limited settings when plasma/serum collection is complex or absent. However, the cut-off values should be optimized when DBS are used for seroprevalence studies. Finally, we recommend carrying out new similar studies with a larger sample size with available paired plasma/DBS specimens per subject and vaccination data, and an equitable number of vaccinated and unvaccinated patients to assign a definitive cut-off value for each pathogen in DBS. The procedures described here could be incorporated into existing data collection protocols in the DRC to monitor vaccinable-preventive disease exposures, herd immunity, and effectiveness of immunization programs.

\section{Declarations}

\section{AUTHOR'S CONTRIBUTIONS}

AH conceptualized and designed the study, looked for funding, coordinated and supervised data collection, contributed to data analysis and result discussion, and critically reviewed the manuscript. ARG, MRG, and AVA collected data, performed the experimental assays and contributed to data analysis and result discussion. ARG and MRG drafted the initial version of the manuscript. AN supervised sample and data collection in Kinshasa, and their transport to Spain. MRD, JCG and GR supervised technical handling in Vircell platform. All authors have read and approved the final manuscript.

\section{ACKNOWLEDGEMENT}

We gratefully acknowledge all patients, their families and local professionals at Monkole and Kalembelembe hospitals in Kinshasa. We want to thank Vircell S.L for free donation of kits and to Silvia Carlos for her support during specimens transport to Spain and her useful comments during data analysis.

\section{COMPETING INTEREST}

All authors declare that they have no conflicts of interest.

\section{References}

1. World Health Organization (WHO). Global Vaccine Action Plan. Monitoring , Evaluation \& Accountability. Anual Report 2020. [Internet][cited 2021 Jun 9] Avalilable from: https://www.who.int/teams/immunization-vaccines-and-biologicals/strategies/global-vaccine-action-plan

2. World Health Organization (WHO).Mejorar la supervivencia y el bienestar de los recién nacidos [Internet]. [cited 2021 Jun 9]. Available from: https://www.who.int/es/news-room/fact-sheets/detail/newborns-reducing-mortality

3. World Health Organization (WHO), UNICEF, World Bank Group, United Nations (UN). Levels and trends in child mortality. [Internet] [cited 2021 Jun 9]. Available from: https://www.unicef.org/reports/levels-and-trends-child-mortality-report-2020

4. UN Inter-agency Group for Child Mortality Estimation (IGME) Democratic Republic of the Congo [Internet] [cited 2021 Jun 9]. Available from: https://childmortality.org/data/Democratic\%20Republic\%20of\%20the\%20Congo

5. UNICEF. Government of the Democratic Republic of Congo doubles its funding for vaccines [Internet] [cited 2021 Jun 9]. Available from: https://www.unicef.org/drcongo/en/press-releases/government-drc-doubles-funding-vaccines

6. UNICEF, USAID. Multiple indicator cluster survey (MICS) Democratic Republic of the Congo 2018. [Internet] [cited 2021 Jun 9]. Available from: https://mics.unicef.org/surveys

7. Medecins Sans Frontieres (MSF) International. DRC: MSF responds to the worrying increase in measles cases [Internet] [cited 2021 Jun 16]. Available from: https://www.msf.org/drc-msf-responds-worrying-increase-measles-cases

8. World Health Organization (WHO). Vaccine-preventable diseases: Monitoring system 2020, global sumary. Country profile. [Internet] [cited 2021 Jun 9]. Available from: https://apps.who.int/immunization_monitoring/globalsummary/countries?countrycriteria\%5Bcountry\%5D\%5B\%5D=COD\&commit=0K

9. Adetokunboh OO, Ndwandwe D, Awotiwon A, Uthman OA, Wiysonge CS. Vaccination among HIV-infected, HIV-exposed uninfected and HIV-uninfected children: a systematic review and meta-analysis of evidence related to vaccine efficacy and effectiveness. Hum Vaccines Immunother. 15(11), 2578-89 (2019).

10. Comité Asesor de Vacunas (CAV-AEP). Vacunación en niños infectados por el virus de la inmunodeficiencia humana (VIH). Manual de vacunas en linea de la AEP [Internet] [cited 2021 Jun 9] Avalilable from: http://vacunasaep.org/documentos/manual/cap-15

11. Department of Health and Human Services. Guidelines for the Prevention and Treatment of Opportunistic Infections in HIV-Exposed and HIV-Infected Children. Section accesed: Preventing vaccine-preventable diseases in children and adolescents with HIV infection. [Internet] [cited 2021 Jun 9 ]. Available at: http://aidsinfo.nih.gov/contentfiles/lvguidelines/oi_guidelines_pediatrics.pdf.

12. Sutcliffe CG, Moss WJ. Do children infected with HIV receiving HAART need to be revaccinated? Lancet Infect Dis. 10(9), 630-42 (2010).

Page $7 / 11$ 
13. Amini F, Auma E, Hsia Y, Bilton S, Hall T, Ramkhelawon L, et al. Reliability of dried blood spot (DBS) cards in antibody measurement: A systematic review. PloS One. 16(3), e0248218 (2021).

14. Su X, Carlson BF, Wang X, Li X, Zhang Y, Montgomery JP, et al. Dried blood spots: An evaluation of utility in the field. J Infect Public Health. 11(3), 373-6 (2018).

15. De Mulder M, Holguín A. [Dried blood spots for monitoring HIV infection in Public Health Programs in developing countries]. Enferm Infecc Microbiol Clin. 31(2), 100-7 (2013).

16. Solomon SS, Solomon S, Rodriguez II, McGarvey ST, Ganesh AK, Thyagarajan SP, et al. Dried blood spots (DBS): a valuable tool for HIV surveillance in developing/tropical countries. Int J STD AIDS. 13(1), 25-8 (2002).

17. Higgins SG, Hoff NA, Gadoth A, Fusellier A, Mukadi P, Alfonso V, et al. Field Test and Validation of the Multiplier Measles, Mumps, Rubella, and VaricellaZoster Multiplexed Assay System in the Democratic Republic of the Congo by Using Dried Blood Spots. mSphere [Internet]. 2019 Aug 14 [cited 2021 Jun 9];4(4). Available from: https://www.ncbi.nlm.nih.gov/pmc/articles/PMC6695516/

18. Krumova S, Golkocheva-Markova E, Angelova S, Voleva S, Pavlova A, Georgieva I, et al. Dried Blood Spots as a Clinical Samples for Laboratory Diagnosis and Surveillance of Vaccine-Preventable Diseases in Bulgaria. Am Sci Res J Eng Technol Sci ASRJETS. 51(1), 183-91 (2019).

19. Fernández McPhee C, Álvarez P, Prieto L, Obiang J, Avedillo P, Vargas A, et al. HIV-1 infection using dried blood spots can be confirmed by Bio-Rad Geenius $^{\text {TM }}$ HIV 1/2 confirmatory assay. J Clin Virol. 63, 66-9 (2015).

20. Rubio-Garrido M, Ndarabu A, Reina G, Barquín D, Fernández-Alonso M, Carlos S, et al. Utility Of POC Xpert HIV-1 Tests For Detection-Quantification Of Complex HIV Recombinants Using Dried Blood Spots From Kinshasa, D. R. Congo. Sci Rep. 9(1), 5679 (2019).

21. Robins EB, Blum S. Hematologic reference values for African American children and adolescents. Am J Hematol. 82(7), 611-4 (2007).

22. Cutts FT, Hanson M. Seroepidemiology: an underused tool for designing and monitoring vaccination programmes in low-and middle-income countries. Trop Med Int Health. 21(9), 1086-98 (2016).

23. Guerra FM, Bolotin S, Lim G, Heffernan J, Deeks SL, Li Y, et al. The basic reproduction number (R0) of measles: a systematic review. Lancet Infect Dis. 17(12), e420-8 (2017).

24. Edmunds WJ, Gay NJ, Kretzschmar M, Pebody RG, Wachmann H, ESEN Project. European Sero-epidemiology Network. The pre-vaccination epidemiology of measles, mumps and rubella in Europe: implications for modelling studies. Epidemiol Infect. 125(3), 635-50 (2000).

25. World Health Organization (WHO). Measles vaccines: WHO position paper. Wkly Epidemiol. Rec. 2017 Apr. 17, $205-228$ [Internet] [cited 2021 Jun 9]. Available at: https://www.who.int/publications-detail-redirect/measles-vaccines-who-position-paper-april-2017

26. World Health Organization (WHO), UNICEF. Democratic Republic of the Congo: WHO and UNICEF estimates of immunization coverage:2019 revision [Internet] [cited 2021 Jun 9]. Available at: https://www.who.int/immunization/monitoring_surveillance/data/cod.pdf

27. Davidkin I, Jokinen S, Broman M, Leinikki P, Peltola H. Persistence of measles, mumps, and rubella antibodies in an MMR-vaccinated cohort: a 20-year follow-up. J Infect Dis. 197(7), 950-6 (2008).

28. Weibel RE, Buynak EB, McLean AA, Roehm RR, Hilleman MR. Persistence of antibody in human subjects for 7 to 10 years following administration of combined live attenuated measles, mumps, and rubella virus vaccines. Proc Soc Exp Biol Med. 165(2), 260-3 (1980).

29. Ashbaugh HR, Cherry JD, Hoff NA, Doshi RH, Alfonso VH, Gadoth A, et al. Measles antibody levels among vaccinated and unvaccinated children 659 months of age in the Democratic Republic of the Congo, 2013-2014. Vaccine. 38(9), 2258-65 (2020).

30. Niewiesk S. Maternal Antibodies: Clinical Significance, Mechanism of Interference with Immune Responses, and Possible Vaccination Strategies. Front Immunol. 5, 446 (2014)

31. Haralambieva IH, Kennedy RB, Ovsyannikova IG, Whitaker JA, Poland GA. Variability in Humoral Immunity to Measles Vaccine: New Developments. Trends Mol Med. 21(12), 789-801 (2015).

32. Holzmann H, Hengel H, Tenbusch M, Doerr HW. Eradication of measles: remaining challenges. Med Microbiol Immunol. 205(3), 201-8 (2016).

33. Whittle H, Aaby P, Samb B, Cissé B, Kanteh F, Soumaré M, et al. Poor serologic responses five to seven years after immunization with high and standard titer measles vaccines. Pediatr Infect Dis J. 18(1), 53-7 (1999).

34. World Health Organization (WHO). Immunological basis for immunization series. Section accesed: Module 7: Measles. [Internet] [cited 2021 Jun 14]. Available at: https://www.who.int/publications/i/item/9789241516655

35. Tejiokem MC, Gouandjika I, Béniguel L, Zanga M-CE, Tene G, Gody JC, et al. HIV-infected children living in Central Africa have low persistence of antibodies to vaccines used in the Expanded Program on Immunization. PloS One. 2(12), e1260 (2007).

36. Moss WJ, Clements CJ, Halsey NA. Immunization of children at risk of infection with human immunodeficiency virus. Bull World Health Organ. 81(1), 6170 (2003).

37. Rubio-Garrido M, González-Alba JM, Reina G, Ndarabu A, Barquín D, Carlos S, et al. Current and historic HIV-1 molecular epidemiology in paediatric and adult population from Kinshasa in the Democratic Republic of Congo. Sci Rep. 10(1), 18461 (2020).

38. Rubio-Garrido M, Reina G, Ndarabu A, Rodriguez-Galet A, Valadés-Alcaraz A, Barquín D, et al. High drug resistance levels could compromise the control of HIV infection in paediatric and adolescent population in Kinshasa, the Democratic Republic of Congo. PloS One. 16(4), e0248835 (2021).

39. Rubio-Garrido M, Avendaño-Ortiz J, Ndarabu A, Rubio C, Reina G, López-Collazo E, et al. Dried Blood Specimens as an Alternative Specimen for Immune Response Monitoring During HIV Infection: A Proof of Concept and Simple Method in a Pediatric Cohort. Front Med. 8, 678850 (2021)

\section{Figures}

Page 8/11 


\section{A}

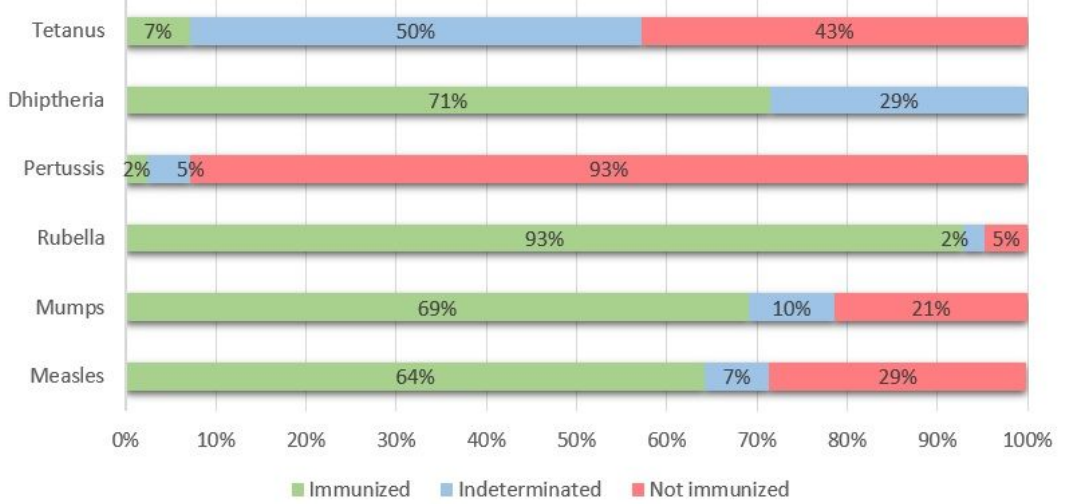

B

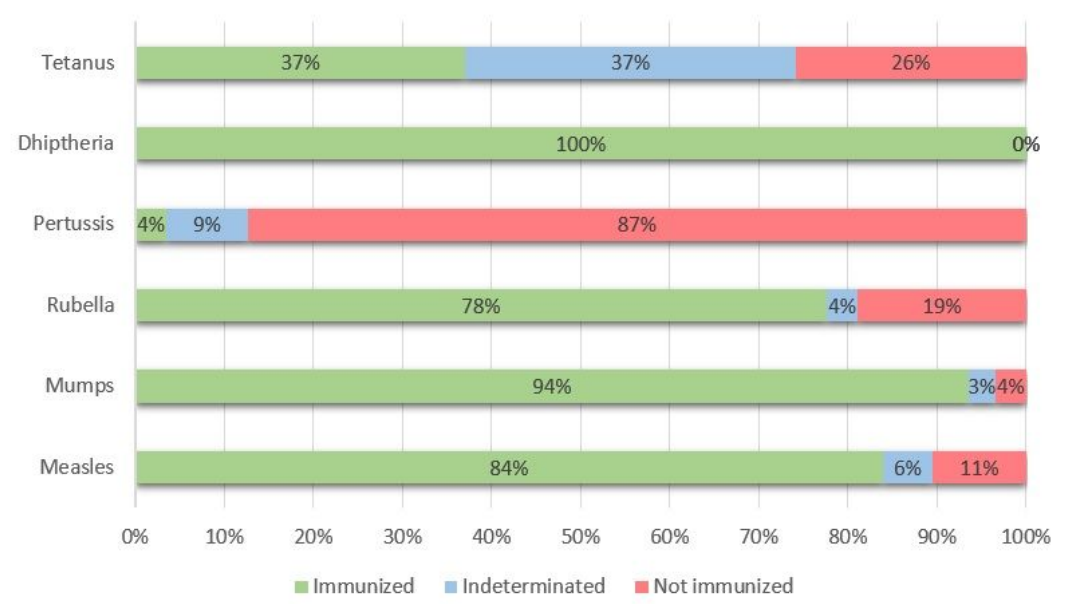

\section{Figure 1}

Percentage of immunization of the 42 paediatric and adolescents from Kinshasa with available plasma (A) or DBS considering the Vircell cut-off (B).

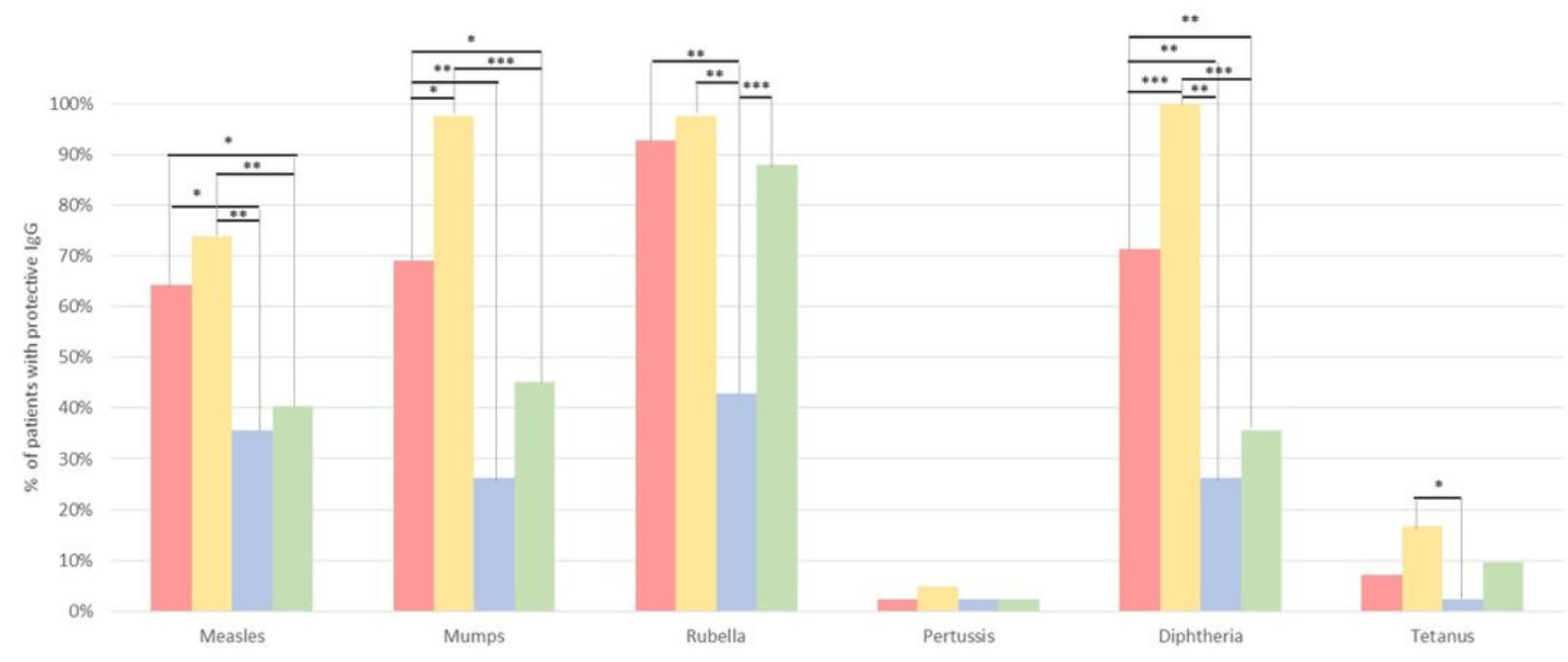

\begin{tabular}{|c|c|c|c|c|c|c|c|c|}
\hline Options & Sample & Cut-off & Measles & Mumps & Rubella & Pertussis & Diphtheria & Tetanus \\
\hline A & 42 Plasma & Vircell & $64.3 \%$ & $69.1 \%$ & $92.9 \%$ & $2.4 \%$ & $71.4 \%$ & $7.1 \%$ \\
\hline B & 42 DBS & Vircell & $73.8 \%$ & $97.6 \%$ & $97.6 \%$ & $4.8 \%$ & $100.0 \%$ & $16.7 \%$ \\
\hline C & 42 DBS & max. Esp. & $35.7 \%$ & $26.2 \%$ & $42.9 \%$ & $2.4 \%$ & $26.2 \%$ & $2.4 \%$ \\
\hline D & 42 DBS & optimum & $40.5 \%$ & $45.2 \%$ & $88.1 \%$ & $2.4 \%$ & $35.7 \%$ & $9.5 \%$ \\
\hline
\end{tabular}


Figure 2

Percentage of immunization in 42 children and adolescents from Kinshasa with paired plasma/DBS considering different cut-offs. The plasma cut-off provided by VirCell for each pathogen is shown in Table 1. The optimal or maximum specificity cut-offs calculated for each pathogen is indicated in Table 3 . Statistically significant differences found: ${ }^{*} \mathrm{p}=0.01-0.03 ; * * \mathrm{p}=0.001-0.003 ;{ }^{* * *} \mathrm{p}<0.0001$.

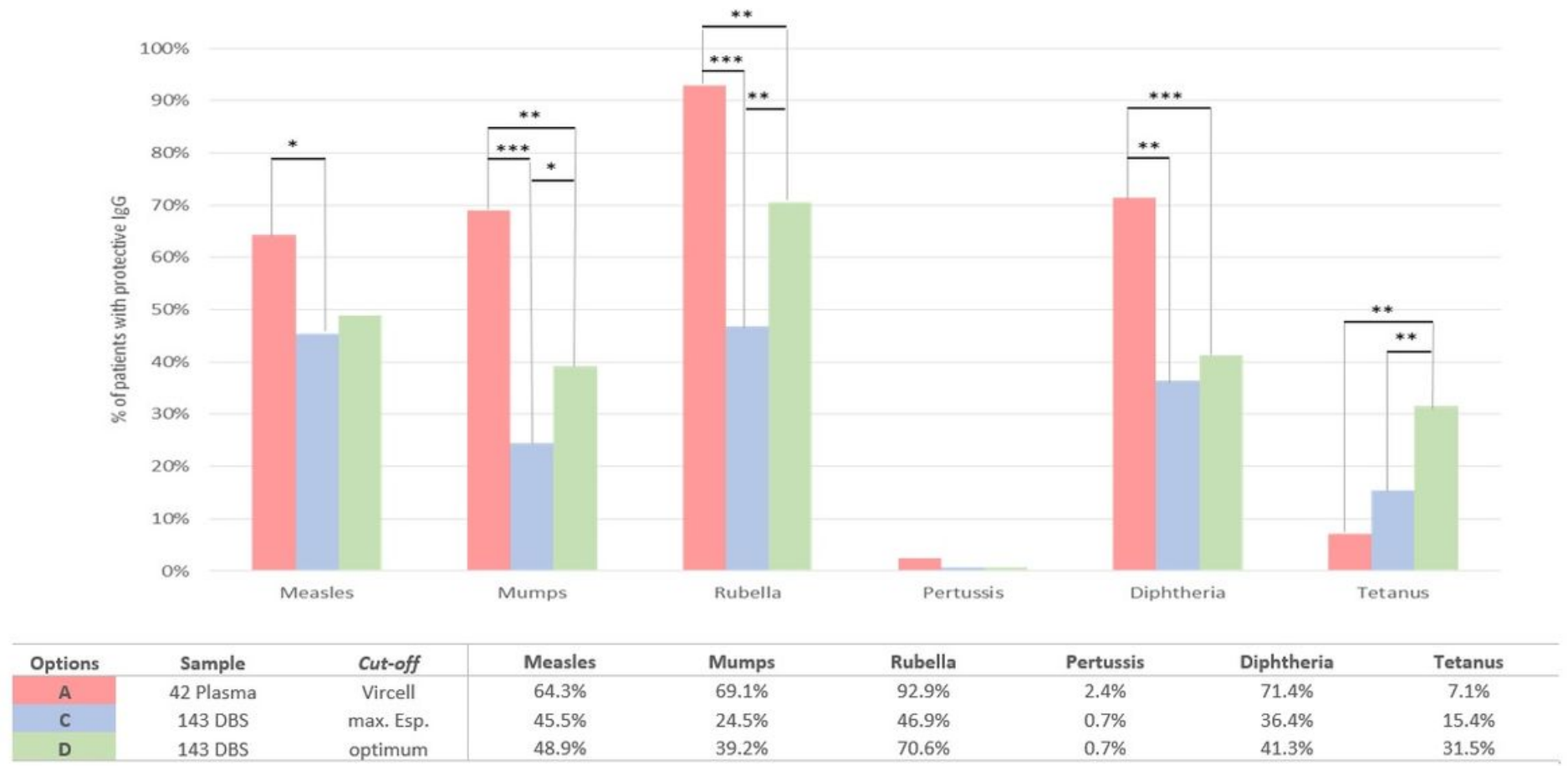

\section{Figure 3}

Comparison of the percentage of immunized patients in the study population (42 plasmas vs. 143 DBS) using the optimal and maximum specificity cut-offs. The plasma cut-off provided by Vircell for each pathogen is shown in Table 1. The optimal or maximum specificity cut-offs calculated for each pathogen is indicated in Table 3. Statistically significant differences found: ${ }^{*} p=0.01-0.03 ; * * p=0.001-0.003 ; * \star * p<0.0001$. 


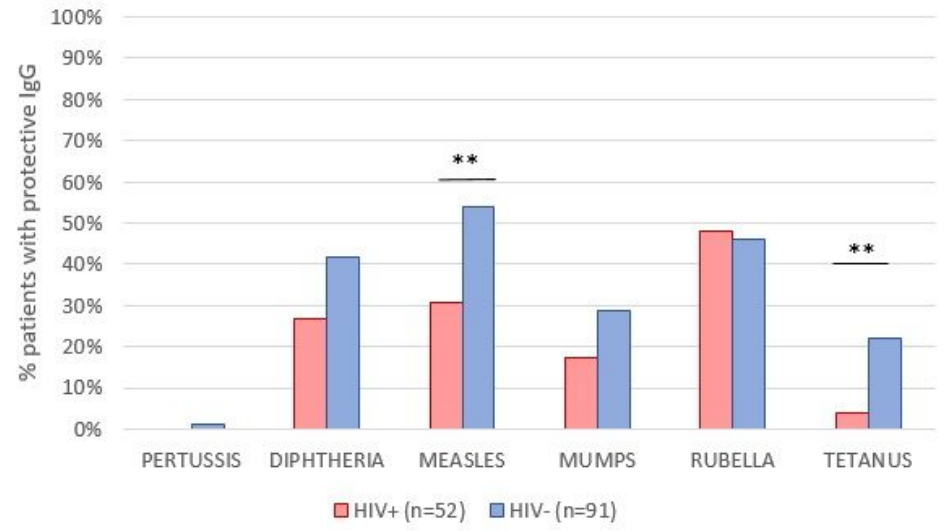

B

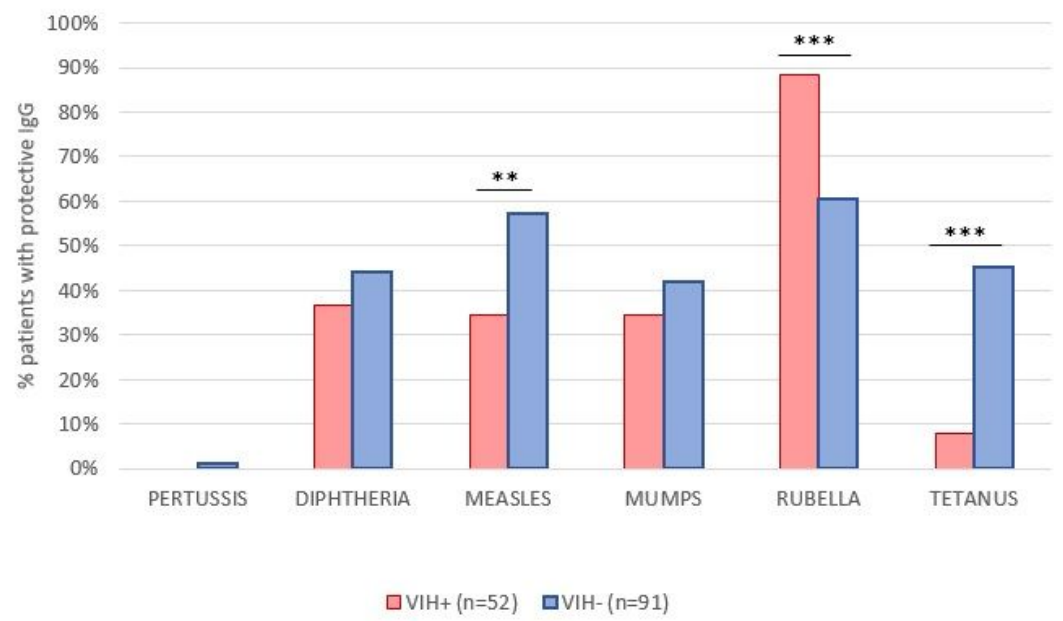

\section{Figure 4}

Percentage of patients immunized against the six pathogens according to the infection or not for HIV in 143 children and adolescents using DBS and cut-off of maximum specificity (A) and optimal (B). HIV+, HIV-infected patients; HIV-, HIV-uninfected patients. With asterisks, statistically significant differences found: ${ }^{* *} p=0.001, * * * p=0.0001$. 